\title{
Prefrontal Neurons of Opposite Spatial Preference Display Distinct Target Selection Dynamics
}

\author{
Therese Lennert ${ }^{1}$ and Julio C. Martinez-Trujillo ${ }^{2}$ \\ ${ }^{1}$ Department of Neurology and Neurosurgery, Montreal Neurological Institute, Montreal, Quebec, H3A 2B4, Canada, and ${ }^{2}$ Department of Physiology, \\ Faculty of Medicine, McGill University, Montreal, Quebec, H3G 1Y6 Canada
}

Neurons in the primate dorsolateral prefrontal cortex (dlPFC) of one hemisphere are selective for the location of attended targets in both visual hemifields. Whether dlPFC neurons with selectivity for opposite hemifields directly compete with each other for target selection or instead play distinct roles during the allocation of attention remains unclear. We explored this issue by recording neuronal responses in the right dlPFC of two macaques while they allocated attention to a target in one hemifield and ignored a distracter on the opposite side. Forty-nine percent of the recorded neurons were target location selective. Neurons selective for contralateral targets (58\%) systematically discriminated targets from distracters faster than neurons selective for ipsilateral targets (42\%). Additionally, during trials in which sensory stimulation remained the same but both stimuli were task irrelevant and animals were required to detect a change in the color of a fixation spot, contralateral neurons still reliably discriminated the putative target from the distracter, whereas ipsilateral neurons did not. The latter result indicates that target-distracter discrimination by contralateral neurons could occur independently of discrimination by ipsilateral cells; thus, the two cell types may represent two different components of the prefrontal circuitry underlying the allocation of attention to targets in the presence of distracters. Moreover, the response of both contralateral and ipsilateral neurons to a single target was substantially reduced by the presence of a distracter in the contralateral hemifield. This result suggests that the presence of the distracter triggered inhibitory interactions within the dlPFC circuitry that suppressed responses to the attended target.

\section{Introduction}

Many neurons in the primate dorsolateral prefrontal cortex (dlPFC) selectively respond to attended targets over unattended distracters (Boussaoud and Wise, 1993; di Pellegrino and Wise, 1993; Rainer et al., 1998; Everling et al., 2002; Lennert and Martinez-Trujillo, 2011). Different from other attention-related brain areas, such as lateral intraparietal area (LIP) and the frontal eye field in which neurons in one hemisphere are predominantly selective for targets in the contralateral visual hemifield (Goldberg and Bushnell, 1981; Blatt et al., 1990; Ben Hamed et al., 2001; Thompson et al., 2005; Patel et al., 2010), dlPFC neurons of one hemisphere are selective for targets in both hemifields. Previous studies have reported that $\sim 60 \%$ of dlPFC neurons prefer contralateral targets, whereas $\sim 40 \%$ prefer ipsilateral targets (Boch and Goldberg, 1989; Funahashi et al., 1989, 1990; Sakagami and Niki, 1994; Rainer et al., 1998; Everling et al., 2002). The roles of dlPFC contralateral and ipsilateral neurons in the processes underlying allocating attention remain unclear.

Received Nov. 5, 2012; revised April 18, 2013; accepted April 27, 2013.

Author contributions: T.L. and J.C.M.-T. designed research; T.L. performed research; T.L. analyzed data; T.L. and J.C.M.-T. wrote the paper.

This work was supported by grants from the Canadian Institutes of Health Research (CIHR), the Natural Sciences and Engineering Research Council of Canada, the EJLB Foundation, and the Canada Research Chair program (all to J.C.M.-T.) and the (IHR Canada Graduate Vanier Scholarship (T.L.).

Correspondence should be addressed to Dr. Julio Martinez-Trujillo, McGill University, Department of Physiology, McIntyre Medical Sciences Building, 3655 Promenade Sir William Osler, Room 1223, Montreal, Quebec, H3G 1Y6, Canada. E-mail: julio.martinez@mcgill.ca.

DOI:10.1523/JNEUROSCI.5156-12.2013

Copyright $\odot 2013$ the authors $\quad 0270-6474 / 13 / 339520-10 \$ 15.00 / 0$
It is possible that the dlPFC of each hemisphere possesses a complete map of the visual field (Funahashi et al., 1989); thus, during the allocation of attention neurons holding representations of targets and distracters located in opposite hemifields may compete through inhibitory interactions (Goldman-Rakic, 1995). One caveat of this hypothesis is that it implies two redundant target-distracter discrimination processes within the right and left dlPFCs during the allocation of attention. Alternatively, neurons of opposite spatial preference within one hemisphere may serve different roles in allocating attention. For example, the dlPFC is extensively interconnected with parietal and temporal cortices (Pandya and Kuypers, 1969; Jones and Powell, 1970; Jacobson and Trojanowski, 1977a,b; Barbas and Mesulam, 1981; Petrides and Pandya, 1984; Goldman-Rakic and Porrino, 1985; Barbas et al., 1991); histological work has reported alternating columns of inputs into the dlPFC of one hemisphere from the ipsilateral parietal and contralateral prefrontal cortex (GoldmanRakic and Schwartz, 1982). It is possible that neurons preferring contralateral and ipsilateral targets are differentially connected. For example, contralateral-preferring neurons of both hemispheres may receive (indirect) inputs from sensory cortices and engage in competitive interactions during target-distracter discrimination through interhemispheric connections; ipsilateral neurons may be involved in processes distinct from the initial target-distracter discrimination, e.g., sending feedback signals to sensory cortices to influence the processing of target and distracter stimuli.

We investigated the role of dlPFC neurons of opposite spatial preference on the allocation of attention to targets in the presence 
of distracters. We recorded responses of dlPFC contralateral and ipsilateral neurons during a task that required covertly attending to a target in the presence of a distracter in the opposite hemifield. We also recorded responses of the same neurons during two variations of the task: (1) when both stimuli were irrelevant and the animals detected a color change in the fixation spot, and (2) when only the target was shown in the absence of distracters. Finally, we recorded the responses of a group of middle temporal area MT neurons in one animal during the same tasks.

\section{Materials and Methods}

Animals. Two young adult male monkeys (Macaca mulatta; monkey Ra, $7 \mathrm{~kg}$; monkey Se, $9 \mathrm{~kg}$ ) participated in the experiments. During the training and testing periods, the animals received fruit juice as reward for correctly performing the task. All procedures complied with the Canadian Council of Animal Care guidelines and were approved by the McGill animal care committee.

Visual stimuli. The stimuli were back-projected on a screen using a video projector (NEC WT610, $1024 \times 768$ pixels resolution, $85 \mathrm{~Hz}$ ) and custom-made software running on an Apple G4 Power PC. The animals viewed the screen at a distance of $57 \mathrm{~cm}\left(1 \mathrm{~cm}=1^{\circ}\right.$ of visual angle $)$. The random dot patterns (RDPs) were generated by plotting colored dots ( white $=13 \mathrm{~cd} / \mathrm{m}^{2}$, gray $=1.9 \mathrm{~cd} / \mathrm{m}^{2}$, pink $=5.4 \mathrm{~cd} / \mathrm{m}^{2}$, green $=0.9$ $\mathrm{cd} / \mathrm{m}^{2}$, blue $=1.58 \mathrm{~cd} / \mathrm{m}^{2}$, red $=0.6 \mathrm{~cd} / \mathrm{m}^{2}$, turquoise $=8 \mathrm{~cd} / \mathrm{m}^{2}$ ) on a dark background (black-gray $=0.02 \mathrm{~cd} / \mathrm{m}^{2}$ ) with a density of three dots per square degree within a circular stationary virtual aperture. All dots within one RDP moved coherently at a speed of $15^{\circ}$ s and were replotted at the opposite side when they crossed the border of the aperture. The radius of the aperture was $4^{\circ}$, and its center was $8^{\circ}$ away from the fixation spot. Two motion directions were chosen: in half of the trials, the dots moved upward $\left(0^{\circ}\right)$ or downward $\left(180^{\circ}\right)$. Brief changes in the motion direction of the dots were introduced as response events and lasted 176 $\mathrm{ms}$ with $32^{\circ}$ intensity clockwise from the current direction.

When recording activity of neurons in area MT, the position, size, and speed of the RDPs were adjusted to match the preferences of the cell. Motion directions of the stimuli were chosen as the preferred direction of the cell and its $180^{\circ}$ opposite.

Target-distracter task. The animals started a trial by pressing a button and keeping gaze within a circular window of $2^{\circ}$ diameter centered on a small fixation spot $\left(0.06^{(0) 2}\right)$. Two moving RDPs were presented at equal distance to the left and right of the central fixation spot. After a variable interval, from 294 to $646 \mathrm{~ms}$ after the onset of the RDPs, the dots in each pattern changed to a different color (e.g., in one pattern to red and in the other to blue). The task for the animals was to select and covertly attend to one RDP (the target) while ignoring the other (the distracter), wait for a brief motion direction change in the target, and release the button within 100-650 ms from the change onset. Target direction changes could occur within a time window ranging from 752 to $2940 \mathrm{~ms}$ after color cue onset. To correctly select the target, the animal learned over several training sessions preceding the recordings a color-rank selection rule (gray $<$ pink $<$ green $<$ blue $<$ red $<$ turquoise; for details, see Lennert and Martinez-Trujillo, 2011). Each correctly performed trial was rewarded with a drop of juice.

To guarantee that the animal correctly selected the target, on half of the trials, the distracter pattern located in the opposite visual hemifield changed direction. The monkey had to ignore this distracter change and wait for the target change. Trials in which the monkey responded to the distracter change (false alarms), or did not respond to the target change within the reaction time window (misses), or broke fixation before the target change onset (fixation breaks), were terminated without reward. The different trial types were presented in random sequence. Only correctly performed trials were included in the analysis. Because of limitations in the number of trials that the animals performed during one recording session, we tested only four different colors at a time (instead of six).

Single-stimulus task. In many dlPFC cells, the single-stimulus task (SST) was tested. After fixation spot onset, a single moving RDP was presented. To assess the responses of the cells to different stimulus attributes, we varied its color [the four colors used in the target-distracter task and white], motion direction (up/down), and location (left/right of the fixation spot). The animals had to release the button in response to a motion direction change in the RDP that occurred randomly between 400 and $2000 \mathrm{~ms}$ after stimulus onset.

Fixation task. We also included trials in which sensory stimulation was identical to that in TDT trials but the RDPs were irrelevant to the animal. A slightly enlarged fixation point $\left(0.167^{(0) 2}\right)$ at trial onset indicated a fixation task (FIX) trial. The timing of the stimuli, color cue, and response event onsets were identical to TDT trials. However, no target and/or distracter change occurred; instead, the animal was required to release the button during detection of a small color change (from white to light gray) in the fixation spot. During a recording session, the monkeys performed half as many FIX trials as TDT trials. Both trial types were randomly interleaved.

Behavioral data were obtained in 46 dlPFC recording sessions for monkey Se and 57 for monkey Ra. Hit rates were calculated as number of hits divided by number of trials.

Surgical procedures and electrophysiological recordings. The animals were implanted with titanium head posts and Cilux recording chambers (Crist Instruments). In both animals, the recording chamber was implanted on top of a circular craniotomy ( $20 \mathrm{~mm}$ diameter) of the frontal bone that provided access to the right prefrontal cortex, to the region anterior to the arcuate sulcus, posterior, and around the sulcus principalis (Lennert and Martinez-Trujillo, 2011). The center of the chamber was positioned at the center of the craniotomy; its stereotactic coordinates were $24 \mathrm{~mm}$ anterior and $17 \mathrm{~mm}$ lateral in Ra, and $30 \mathrm{~mm}$ anterior and $17 \mathrm{~mm}$ lateral in Se. The chambers were circular, $20 \mathrm{~mm}$ in diameter with $20^{\circ}$ and $35^{\circ}$ base angle, respectively. In monkey Ra, the chamber was positioned with a lateral tilt of $12^{\circ}$ from the vertical, and in monkey Se, the chamber was positioned parallel to the vertical. In the anteroposterior plane, both chambers were parallel to the vertical (the vertical and the horizontal planes were defined in stereotactic coordinates).

We recorded from the right dlPFC of both animals. During each experimental session, transdural penetrations were made with standard epoxy-insulated extracellular tungsten electrodes (shank diameter, 500 $\mu \mathrm{m}$; impedance, $2-4 \mathrm{M} \Omega$ at $1 \mathrm{kHz} ; \mathrm{FHC}$ ). A guide tube positioned at 5-10 $\mathrm{mm}$ distance from the recording electrode, touching but not penetrating the dura, served as the reference. We used a Plexon data acquisition system (MAP) to record and store spike and local field potential (LFP) data simultaneously. The electrode signal was passed through a head stage with unit gain and then split into the spike and the LFP components. For spike recordings, the signal was filtered between 250 and $8000 \mathrm{~Hz}$, amplified, and digitized at $40 \mathrm{kHz}$. Single-unit spiking activity was then isolated using Plexon online and offline sorting software.

In monkey Se, we also collected data during 21 recording sessions from single neurons in area MT of the left hemisphere. Transdural penetrations were made with guide tubes through a chamber implanted on top of a craniotomy of the parietal bone and providing access to area MT. Spikes were recorded using extracellular tungsten electrodes (1-2 M $\Omega$ at $1 \mathrm{kHz}$; FHC).

Data analyses. Analysis of spike data (firing rates) and statistical tests were performed using MATLAB (MathWorks). The activity/response of each neuron was plotted as a spike density function, generated by convolving a spike train with a function that resembled a postsynaptic potential, i.e., the time constant of the growth phase and the time constant of the decay phase were 1 and $20 \mathrm{~ms}$, respectively (Murthy et al., 2007). The mean firing rate at different stages of the task was analyzed by computing the mean over a given epoch of the trial-averaged spike density function. When indicated, single-trial firing rates of each neuron were normalized to the mean activity within a $300 \mathrm{~ms}$ time window before color cue onset (TDT) or stimulus onset (SST).

Mutual information. We assessed single-neuron selectivity to the target location using a mutual information (MI) approach (Shannon and Weaver, 1949; Buschman and Miller, 2009). The MI statistic reflects how well target location can be predicted based on the firing rate of a given neuron. The information carried by neurons was calculated using the following:

$$
\mathrm{M} I(r, s)=\sum_{r, s} P(r, s) \log \frac{P(r, s)}{P(r) P(s)},
$$


A
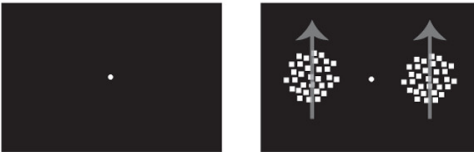

RDPs onset

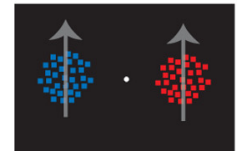

color cue
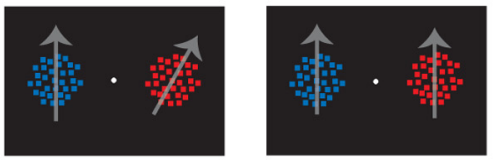

transient RDP change

$0 \mathrm{~ms}$

$350 \mathrm{~ms}$

$650-1000 \mathrm{~ms}$

$1750-3940 \mathrm{~ms}$

response

and juice reward

B
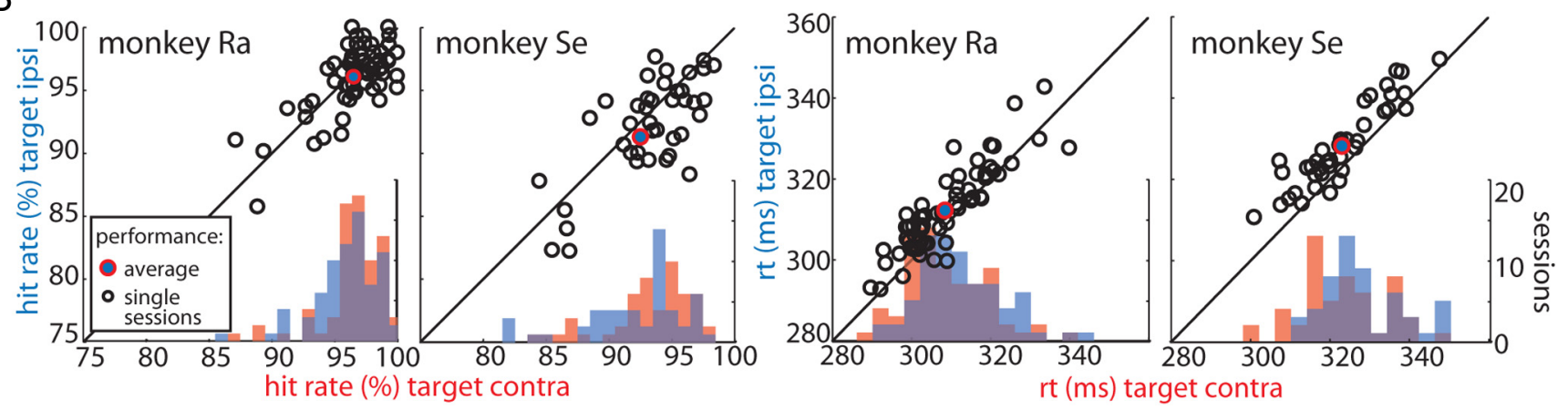

Figure 1. Task and behavioral performance. $A$, TDT trial sequence. Black panels represent the screen across the duration of the example trial. The target and distracter stimuli were defined using a color-rank rule (for details, see Materials and Methods). Gray arrows indicate the RDPs motion direction and are for visualization purposes only. $\boldsymbol{B}$, Hit rates and reaction times (rt) as a function of target position (red, target contralateral; blue, target ipsilateral) for both animals. Black open circles represent single-session performances during TDT. The solid blue-red circles indicate average performances across sessions during TDT.

where $s$ is the target location, $r$ is the response, and the log is base 2. In our case, MI was calculated using two target locations and eight bins for the neural response (Musallam et al., 2004). To determine whether and when the observed level of MI was significantly different from chance, we applied a randomization test whereby the association between firing rate and target location was randomly shuffled and the amount of MI was recalculated. By repeating this process 5000 times, a null distribution was constructed that was then compared with the actual MI.

MI was computed in windows of $25 \mathrm{~ms}$ and in steps of $10 \mathrm{~ms}$ along the time course of the trial. Significance was determined within each bin and considered significant only if the amount of MI exceeded $95 \%$ of the null distribution ( $p<0.05$ in each bin). The point when an individual neuron began to significantly reflect the target location was defined as the time point at which significant MI was obtained in at least three consecutive bins (chance level $=0.05^{3}=0.00125$ ). Based on those individual selection latencies, we created latency distributions for the different populations of neurons.

To determine when a population as a whole began to represent the target location, we compared the observed MI within each bin with zero using Wilcoxon's signed-rank tests, evaluated at $p<0.05$. Selection latency was defined as the time point when the population MI was significant in at least five consecutive bins.

Within tasks, the number of trials of contralateral and ipsilateral targets obtained for each cell was identical. Approximately 120 trials were available during TDT and $\sim 60$ during FIX. During SST, we considered only neurons with 50 or more trials per target location. For direct comparison of the MI time course of a cell during SST and TDT, the amount of MI during TDT was recomputed using a downsampled number of random trials that matched the number of trials available during SST. We repeated this 50 times and used the average downsampled MI for comparison.

Signal detection analyses. Because the MI statistic only indicates the strength of the target location selectivity but does not indicate its direction (i.e., whether a cell prefers contralateral or ipsilateral targets), in addition we applied a receiver operator characteristic (ROC) analysis (Thompson et al., 1996). A derived measurement, the area under the ROC curve (auroc), represents the probability with which, on the basis of firing rates, an ideal observer can reliably identify the target in the pres- ence of a distracter. A value of 0.5 indicates that a given firing rate could have been elicited with equal probability by the target at either location. A value of 1.0 indicates that responses to the contralateral target were always greater than responses to the ipsilateral target. Conversely, a value of 0 indicates that responses to the ipsilateral target were always greater than responses to the contralateral target.

The auroc values for individual neurons were calculated for a $50 \mathrm{~ms}$ window slid in steps of $1 \mathrm{~ms}$ along the spike train. A neuron was classified as contralateral or ipsilateral based on its average auroc value during the $100 \mathrm{~ms}$ around its individual selection latency as identified using the MI statistic. For each single cell, the maximum selection performance was obtained (value of $>0.5$ for contralateral cells; value of $<0.5$ for ipsilateral cells) and used as input to the Hartigan's dip test, which assessed bimodality in the obtained distribution of maximum auroc values.

Fano factor. The Fano factor was computed in $50 \mathrm{~ms}$ bins, slid along the trial in $1 \mathrm{~ms}$ increments. The spike count was obtained in each bin for each trial. For each bin, the Fano factor was computed as the ratio of variance in spike counts across trials to spike count mean. Significant differences in Fano factor between contralateral and ipsilateral cells were assessed using Wilcoxon's rank-sum tests for each bin along the trial. Significance was evaluated at $p<0.05$ during at least 10 successive bins.

Task selectivity index. To quantify differences in the amount of MI between SST and TDT, we computed a task selectivity index (TSI) $\left[\mathrm{TSI}_{\mathrm{MI}}=\left(\mathrm{MI}_{\mathrm{SST}}-\right.\right.$ $\left.\left.\mathrm{MI}_{\mathrm{TDT}}\right) /\left(\mathrm{MI}_{\mathrm{SST}}+\mathrm{MITDT}\right)\right]$. This yielded the TSIs in bins of $25 \mathrm{~ms}$ across time and allowed us to compare modulation across the two tasks for the neuronal groups of interest. TSI values of 0 indicate similar MI during SST and TDT; indices between 0 and 1 indicate an enhancement of MI during SST relative to responses during TDT, whereas values between 0 and -1 point to a relative decrease. Note that we used a downsampled version of the MI during TDT to match the number of trials available during SST (for details, see above).

\section{Results}

\section{Behavioral performance}

Two macaque monkeys were trained in a task that involved directing attention to a target in the presence of a distracter (TDT; Fig. $1 A$; see Materials and Methods). A task trial was initiated when the animal 
A

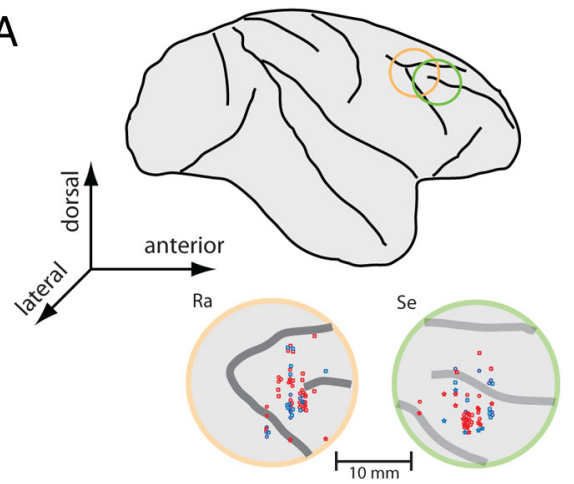

B

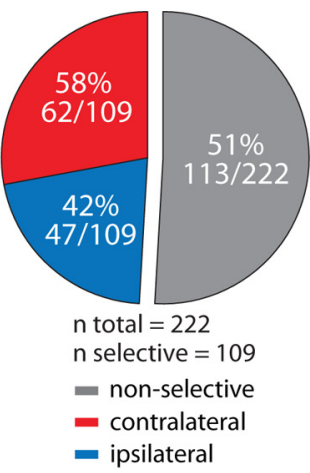

C
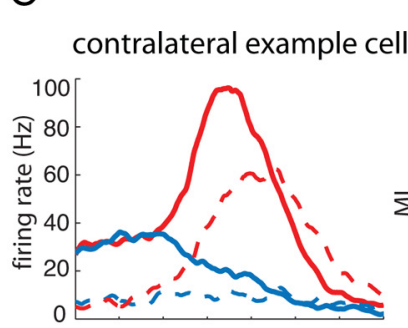

ipsilateral example cell
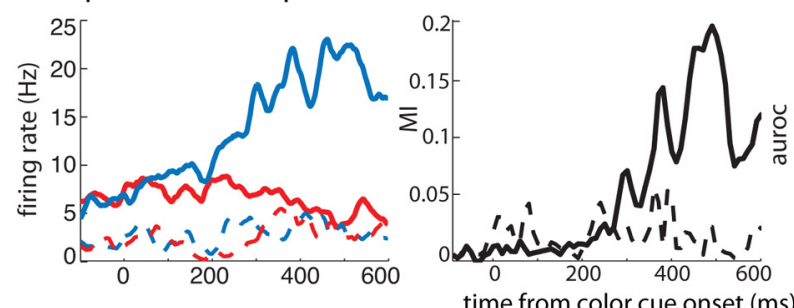

time from color cue onset (ms)
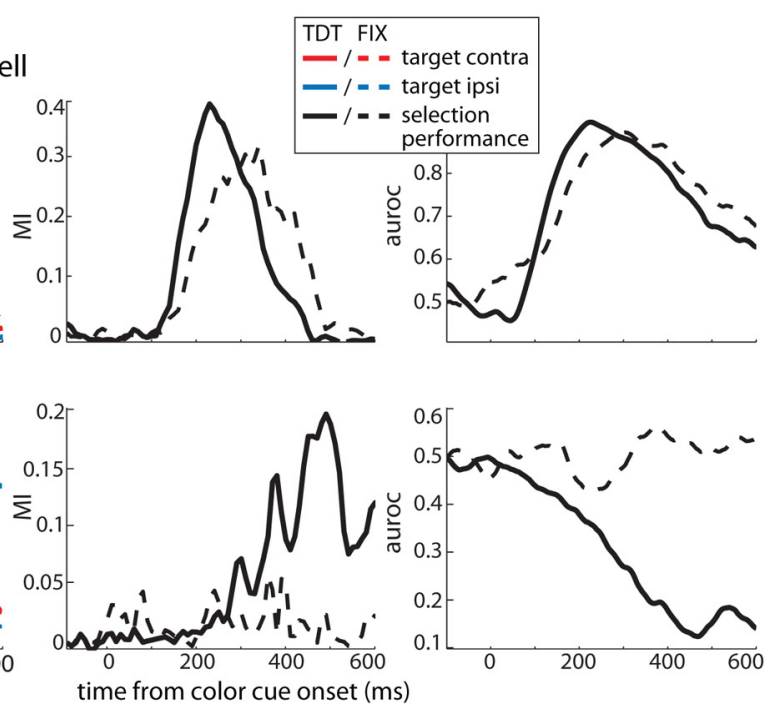

Figure 2. Recording sites and neuronal selectivity. $\boldsymbol{A}$, Lateral view of the macaque brain with chamber locations indicated (top) and recording sites (bottom) in monkeys Ra and Se, respectively. Red and blue markers indicate the location of units preferring targets contralaterally and ipsilaterally, respectively. $\boldsymbol{B}$, Classification of dIPFC cells based on their MI into target location selective and nonselective (gray); selective cells were further classified into contralateral (red) and ipsilateral (blue) preferring based on their auroc values. C, Two single-cell examples. Left, Firing rates as a function of time to contralateral (red) and ipsilateral (blue) targets during TDT (solid lines) and FIX (dashed lines) from color cue onset. Middle and right, The corresponding selection performance of the cells (MI and auroc, respectively) across time during TDT (solid black lines) and FIX (dashed black line).

that the RDPs were irrelevant. The animals were required to release the button in response to a small color change in the fixation spot. Although during FIX neither of the RDPs was the target, we used the same color combinations as during TDT. Thus, for each pair of stimuli during TDT, there was a matching identical pair during FIX. In some of the following analyses, we defined the putative target during FIX relative to the TDT. Overall behavioral performances of both monkeys in the SST and FIX tasks were close to $100 \%$. Effects of target position were absent in both animals during FIX ( $p>0.15$, Wilcoxon's signed-rank tests; data not shown).

These data demonstrate that the animals were proficient in all tasks and performed well above chance level. The effects of target position in both animals' reaction times may indicate advantages in motor preparation for stimuli on the left side. Importantly, the data analysis in the following sections focused on neuronal responses preceding direction changes in the target and distracter. This ensured that any response modulation was attributable to the allocation of attention rather than to changes in stimulus direction and exogenous allocation of attention to such changes (Busse et al., 2008) or motorpreparation-related processes (Alexander and Crutcher, 1990).

\section{Neuronal response selectivity and dynamics of target selection}

We recorded the responses of 222 neurons in the right dlPFC of two monkeys (106 in monkey Ra, 116 in monkey Se; Fig. 2A). During the recording sessions, no prese-

directed gaze to a central fixation spot and pressed a button. Then, two white moving RDPs appeared to the left and right of the fixation spot. After a brief delay, the white RDPs changed their colors, indicating which pattern was the target and which one was the distracter. Based on a learned color rule, the animals had to covertly select the target, sustain attention on it, and release the button in response to a transient change in its motion direction while ignoring changes in the distracter. Within 3-5 months of training, both animals reached stable performances in the task. Average hit rates were above $90 \%$ for both animals (Fig. 1B, filled red-blue markers). A comparison of hit rates as a function of target location (contralateral $=$ left, ipsilateral $=$ right) revealed slightly better performances for left targets (Wilcoxon's signed-rank tests, $p=0.0375$ and $p=0.0047$ for monkeys Ra and Se, respectively). In both animals, reaction times were shorter for targets on the left (contralateral) hemifield ( $p<0.00001$, $t$ tests).

In addition, we also tested two variations of the TDT (for details, see Materials and Methods). In the SST, we presented a single moving RDP on the screen to either the left or right of the fixation spot. The animals' task was to release the button in response to a transient motion direction change in the RDP. We also included FIX trials, in which sensory stimulation was identical to that during TDT but a slightly enlarged fixation spot at the beginning of the trial indicated lection of neurons took place, i.e., any neuron active during the trial period was recorded. Offline analysis of neuronal activity revealed that selectivity for both motion direction and color was negligible, with $<5 \%$ of the neurons being selective at $p<0.05$. We thus averaged across motion directions and colors during the following analyses. In a first step, we determined for each neuron the amount of information contained in its firing rate about target location using an MI statistic (Shannon and Weaver, 1949; Buschman and Miller, 2009). MIs were computed in bins of 25 $\mathrm{ms}$ and in increments of $10 \mathrm{~ms}$ during the period from $200 \mathrm{~ms}$ before color cue onset to $600 \mathrm{~ms}$ after. A neuron was considered target location selective if MIs in at least three consecutive bins exceeded the 95th percentile value obtained through a random permutation procedure (for details, see Materials and Methods). The time when this criterion was first met was considered the selection latency of the neuron. Of the 222 neurons, 109 (49\%) were target location selective.

Although MI provides an estimate of how informative the firing of a cell is with regards to target location, it does not provide information about the preferred location of the neuron (i.e., contralateral or ipsilateral in our case). To determine the preferences of the cells, we used signal detection analysis. For each neuron, ROC curves were obtained in bins spanning $50 \mathrm{~ms}$ and in incre- 
ments of $1 \mathrm{~ms}$ during the same trial period. The auroc served as a measure of neuronal performance, i.e., 0.5 indicates chance performance and 0 and 1 perfect discriminability (Thompson et al., 1996). In our configuration, auroc values between 0.5 and 1 indicate that the neuron fired systematically more strongly to contralateral targets, whereas auroc values between 0.5 and 0 indicate that the neuron fired more strongly to ipsilateral targets. To determine the side preference for each of the 109 selective cells, we obtained the average auroc value within the $100 \mathrm{~ms}$ around the individual selection latency of each cell. We found that 62 of 109 (58\%) preferred contralateral targets, and 47 of 109 (42\%) preferred ipsilateral targets (Fig. 2B).

Figure $2 C$ shows the firing rate (left) of two single-cell examples during TDT and FIX along with their selection performance assessed using MI (middle) and auroc (right). During TDT, the contralateral example neuron showed a strong and early $(\sim 100$ $\mathrm{ms}$ ) increase in firing rate to the target stimulus when positioned contralaterally (solid red line), whereas it decreased its response to almost zero when the target was at the ipsilateral position (solid blue line). During FIX (dashed lines), this neuron showed a considerably lower pre-cue firing rate. Surprisingly, a strong response increase to the contralateral target is observed, although in this task no target-distracter discrimination was required. During TDT, the MI increased after $\sim 100 \mathrm{~ms}$ from cue onset to reach a maximum between 200 and $300 \mathrm{~ms}$. During FIX, the increase in MI was similar. Correspondingly, auroc values during both TDT and FIX increased to almost 0.9 at $\sim 200 \mathrm{~ms}$ after cue onset.

The ipsilateral example neuron showed a strong increase in firing rate to ipsilateral targets (solid blue line) during TDT starting at $\sim 200 \mathrm{~ms}$ after cue onset, whereas a slight decrease was observed in response to the contralateral target (solid red line). Similar to the contralateral neuron, it showed a decrease in precue firing rate during FIX trials. Unlike the contralateral neuron, this unit did not modulate its firing rate during FIX when both stimuli were task irrelevant. Correspondingly, the MI increased during TDT after $200 \mathrm{~ms}$ from cue onset to reach a maximum at $500 \mathrm{~ms}$, whereas it remained around zero during FIX. Similarly, auroc values during TDT decreased to a minimum of almost 0.1 at $500 \mathrm{~ms}$, whereas they remained around chance during FIX.

We next tested whether the sample of target-selective neurons consisted of two distinct groups according to their preferred target location. For each target-selective neuron, we determined its maximal selection performance during TDT (maximal MI and maximal/minimal auroc values during 0-700 $\mathrm{ms}$ from color cue onset; Fig. 3). Although MI values were unimodally distributed between 0.35 and 0.65 (histogram on the top; Hartigan's dip test, $\operatorname{dip}=0.02, p=0.976$ ), auroc values were bimodally distributed (histogram on the right; $\operatorname{dip}=0.06, p=0.004$ ). The latter result indicates that our sample of dlPFC target location-selective cells was divided according to their preferred target location into two subgroups: contralateral and ipsilateral neurons.

In the following, we systematically assessed and compared the discrimination performances (MI and auroc) of the two subgroups of neurons. In the remaining of this section, population responses drawn in red refer to the contralateral group and those in blue to the ipsilateral group. The MI time courses of contralateral and ipsilateral cells evolved similarly to that of the example neurons (Fig. 4A). During TDT (top, solid lines), target selection diverged from zero faster in contralateral relative to ipsilateral neurons. Using Wilcoxon's signed-rank tests, we determined the time point at which the population MI was significantly different from zero $(p<0.05)$ in at least five consecutive time bins. Re-

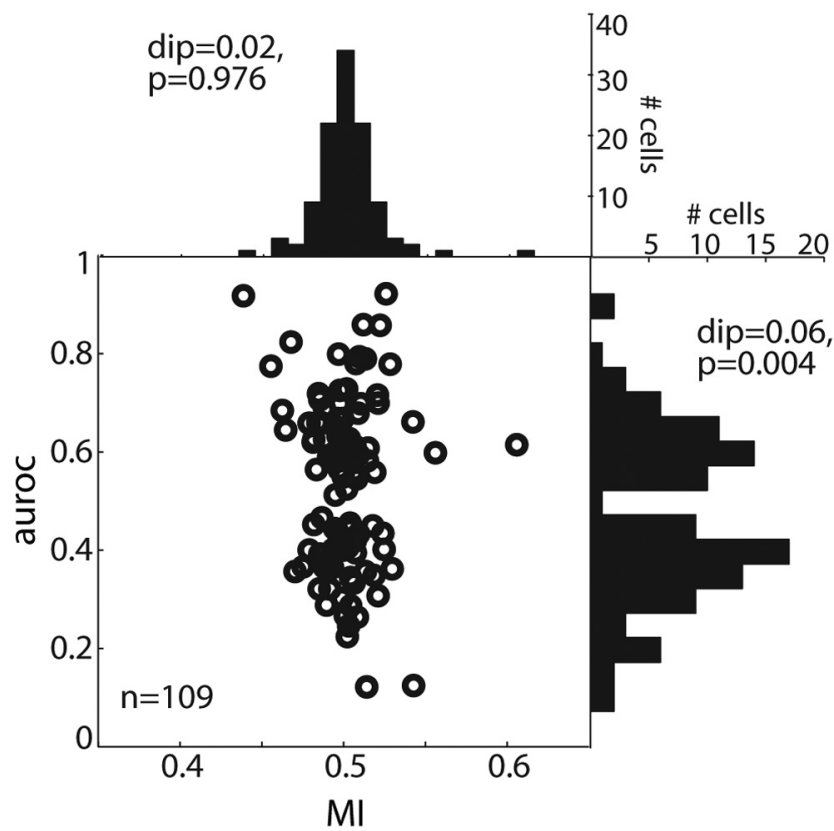

Figure 3. Bimodality. Open black circles represent the maximal MI ( $x$-axis) and maximal auroc ( $y$-axis) values obtained during TDT for each target location-selective cell $(n=109)$. The histogram on top shows the distribution of Mls across the population; the histogram to the right shows the distribution of auroc values. The Hartigan's dip test assessed the bimodality of the distributions (MI, dip $=0.02, p=0.976$; auroc, dip $=0.06, p=0.004$ ).

A
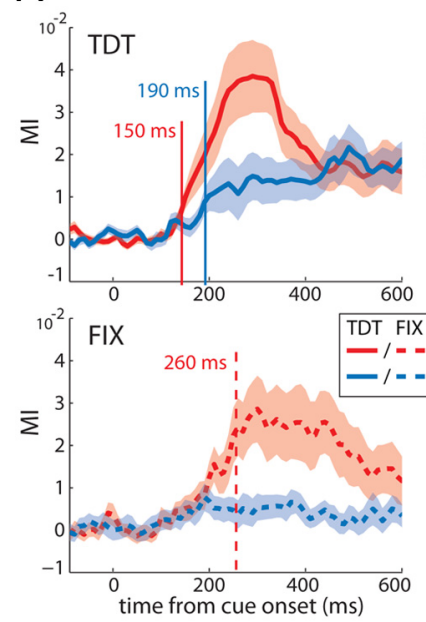

Figure 4. Target selection of contralateral versus ipsilateral groups of cells. $\boldsymbol{A}$, Average Ml as a function of time from cue onset for contralateral (red, $n=62$ ) and ipsilateral (blue, $n=47$ ) populations of cells during TDT (top, solid lines) and FIX (bottom, dashed lines). Red and blue vertical lines represent the time point when the average MI was first significantly different from zero for contralateral and ipsilateral groups, respectively (Wilcoxon's signed-rank tests for significant differences from zero, $p<0.05$ during at least 5 consecutive bins). Note that no latency could be obtained for ipsilateral cells during FIX. Shaded areas represent SEM. B, Average target selectivity assessed using auroc across time. The same color and line coding as in $\boldsymbol{A}$ applies. $\boldsymbol{C}$, Distributions of TDT selection latencies across single contralateral (red) and ipsilateral (blue) cells (Wilcoxon's rank-sum test evaluated at $p<0.05$ ).

spective latencies for contralateral and ipsilateral populations were 150 and $190 \mathrm{~ms}$ from cue onset. During FIX (bottom, dashed lines), the contralateral population selected the target reliably with a latency of $260 \mathrm{~ms}$, whereas ipsilateral cells did not select the target during the entire trial period. The corresponding 
average auroc time courses of contralateral and ipsilateral cells corroborate these distinct selection dynamics (Fig. $4 B$ ).

In addition, we assessed selection latencies at the singleneuron level based on the individual target selection latencies of the cells defined as stated above. Comparison between the two groups of neurons revealed a significantly shorter average latency for contralateral compared with ipsilateral cells during TDT [rank-sum test (mean \pm SEM), $255 \pm 17$ and $330 \pm 22 \mathrm{~ms}, p=$ 0.0056, respectively; Fig. 4C]. During FIX when the stimuli were irrelevant, 25 contralateral cells reached MI values exceeding the 95th percentile obtained from the shuffled permutation distribution. The average selection latency of this subgroup of cells was not significantly different from that of the entire contralateral population during TDT (FIX, $260 \pm 27.9 \mathrm{~ms}$; Wilcoxon's signedrank test, $p>0.05$; data not shown). Of the ipsilateral cell population, only 11 neurons reliably selected the target during FIX. Interestingly, 7 of these 11 cells displayed a switch in their spatial preference during FIX when the stimuli were task irrelevant: they fired more strongly for the target on the contralateral side. Accordingly, with $250 \pm 33 \mathrm{~ms}$, the average selection latency of this subgroup resembled the one observed in the contralateral cell population (data not shown). In comparison, none of the 25 contralateral cells selective during FIX showed a switch in their spatial preference; all maintained their contralateral selectivity. The difference in target-distracter discrimination between contralateral and ipsilateral cells during FIX was not attributable to differences in the animals' behavioral performances between sessions in which we recorded contralateral and ipsilateral neurons (Wilcoxon's rank-sum test, $p=0.09$ ).

We further assessed the maximal amount of information contained in contralateral and ipsilateral cells by comparing the maximal MI values of the cells. This comparison did not reveal significant differences between the populations (rank-sum test, $p>0.05$; data not shown; for the distribution of maximal MIs, see Fig. 3), indicating that the maximal amount of information about target location present in both groups was similar.

The response variability of a neuron can affect how reliably information is encoded in its firing rate. To examine whether the observed differences in target selection dynamics between contralateral and ipsilateral cells may be driven by differences in response variability across time, we computed the Fano factor, the ratio of spike count variance to mean spike count, in $50 \mathrm{~ms}$ bins slid along the trial in $1 \mathrm{~ms}$ steps (data not shown). The Fano factor during all four conditions showed only minor modulations after the color cue onset. A comparison between contralateral and ipsilateral cells revealed no significant differences (Wilcoxon's rank-sum tests, $p>0.05$, evaluated during 10 consecutive bins). These results suggest that differences in response variability between cell groups cannot account for the differences in target selection dynamics.

Finally, we examined the waveform widths of the cells to test the possibility that contralateral and/or ipsilateral cells were associated with a particular type of neuron, e.g., contralateral cells with putative pyramidal cells (broad-spiking) and ipsilateral cells with putative inhibitory neurons (narrow-spiking). Our analysis revealed no consistent pattern: narrow-spiking and broad-spiking neurons occurred intermixed in both groups of cells (data not shown). These results suggest that, in our sample, contralateral and ipsilateral cells cannot unequivocally be assigned to one particular neuronal type; both groups appear to comprise putative inhibitory interneurons as well as putative pyramidal neurons.

In summary, our data show that contralateral neurons discriminated targets significantly faster compared with ipsilateral cells. Moreover, the population of contralateral cells reliably discriminated targets during FIX, when the two RDPs were irrelevant, whereas the population of ipsilateral neurons did not do so.

\section{Target selectivity in the absence versus presence of a distracter}

In 47 of the 109 target location-selective cells, we recorded a sufficient number of trials during the SST condition to assess target location selectivity using MI. Note that, in trials of this condition, only the target was presented on either the left or right visual hemifield at the same positions as during the TDT. In 25 of these 47 cells, MI exceeded the 95th percentile value obtained through the random permutation procedure and thus showed significant target location selectivity during SST. Subsequent evaluation of the auroc time courses of the cells revealed that $84 \%$ $(n=21)$ of these cells responded more strongly to the single RDP positioned on the contralateral side, whereas only $16 \%(n=4)$ preferred the ipsilateral side. These results indicate that, during SST, considerably fewer neurons were selective for the target location relative to the TDT. Thus, the presence of a distracter caused the neurons to "acquire" selectivity for the target location. Most of the neurons that revealed target selectivity only during TDT $(n=22)$ preferred ipsilateral targets (ipsilateral, $n=15$; contralateral, $n=7$ ).

Of the 25 neurons that were target location selective during SST, the majority were contralateral neurons. Surprisingly, we found that a subset of these contralateral SST cells switched their spatial preference from contralateral during SST to ipsilateral during TDT $(n=$ 5 ). We thus classified our cells into three groups: congruent cells that maintained their spatial preference across tasks comprising both contralateral and ipsilateral neurons, and incongruent cells that switched from contralateral during SST to ipsilateral during TDT comprising only contralateral neurons (Fig. 5A).

The fundamental difference between SST and TDT was that, in the latter but not in the former task, a distracter stimulus was presented in the hemifield opposite to the target. To assess how the information about target location encoded by the group of SST target-selective neurons was affected by the presence of the distracter, we computed a selectivity index as a function of time. The TSI was computed as MI during SST minus MI during TDT divided by their sum. A TSI $>0$ indicates that information about the target location decreased in the presence of a distracter, a TSI $<0$ indicates the opposite, and a TSI $=0$ means that the presence of the distracter did not affect the amount of MI. Note that, for each cell, MI during TDT was recalculated using a downsampled number of trials that matched the number of trials available during SST (for details, see Materials and Methods). Figure $5 B$ displays the resulting TSI time courses for the three groups of neurons. All TSIs were positive, indicating that in all cells the amount of information about the stimulus decreased by adding the distracter (during TDT). The TSIs of the two contralateral groups of neurons (red and purple lines) displayed an immediate and sharp increase from zero, whereas that of ipsilateral congruent cells (blue line) increased more slowly and only considerably later. These latency effects are in line with those reported above during TDT: selectivity modulation set in first in contralateral cells, followed by that in ipsilateral cells.

To examine whether these suppressive effects on MI were attributable to a decrease in firing rate in response to targets at the preferred location or alternatively, to an increase in response to the target at the opposite location, we compared the maximal firing rate of each cell during SST and TDT (Fig. $5 C$ ). When the target was positioned at the preferred location of the cell during 
both tasks (i.e., contralateral for contralateral congruent cells, ipsilateral for ipsilateral congruent cells, and contralateral during SST/ipsilateral during TDT for contralateral incongruent cells), individual firing rates were well above the unity line (linear regression, three outliers removed; slope $=2.03 ; r=0.668 ; F$ test, $p=$ $0.0087)$. The same analysis for targets at the opposite side revealed a slope not significantly different from 1 at the Bonferroni's corrected $\alpha$ level of 2.5\% (two outliers removed; slope $=1.22 ; r=0.852$; $F$ test, $p=0.03$ ), indicating that firing rates during SST and TDT were similar.

These findings suggest that introducing a distracter opposite to the target at the preferred location (but not vice versa) triggered inhibitory interactions that resulted in decreased firing rates and, consequently, in reduced $\mathrm{MI}$ about the target location. A similar effect has been observed in studies of attention in visual cortex (Reynolds et al., 1999; Khayat et al., 2010). The limited number of SSTselective cells available to us prevented a more detailed analysis comparing contralateral and ipsilateral cells.

\section{Responses of MT neurons during TDT and FIX}

Previous studies have shown that, during voluntary allocation of attention, neurons in visual areas such as MT differentially respond to targets and distracters inside their receptive fields (RFs) (Martinez-Trujillo and Treue, 2004). To explore how MT neurons behave during the TDT and FIX tasks, we recorded the activity of single neurons $(n=21)$ in left area MT of monkey Se during both tasks. The motion direction and speed of RDPs were chosen to match the preferences of the recorded cell (Treue and Martínez-Trujillo, 1999). We applied the same MI analysis as described above for dlPFC neurons.

Of the 21 units, $15(71 \%)$ were selective for the target location as measured using MI. Figure $6 \mathrm{~A}$ shows the responses of a singlecell example during TDT (solid lines) and FIX (dashed lines). The neuron exhibited a strong response to the onset of the two white RDPs (at approximately $-100 \mathrm{~ms}$ ), which was similar across tasks. During TDT, the cell showed a brief, dip-like decrease in its response after cue onset for targets at both locations. Stereotyped dip-and-rise activity has been reported previously in different tasks and brain areas (Sato and Schall, 2001; Roitman and Shadlen, 2002; Leon and Shadlen, 2003; Huk and Shadlen, 2005; Li et al., 2006), and it has been suggested to reflect a "reset" at the beginning of cognitive processes (Huk and Shadlen, 2005). This initial dip was followed by a sharp increase to values around the prestimulus baseline. At $400 \mathrm{~ms}$ after the cue onset, responses to contralateral (black line) and ipsilateral (gray line) targets started to diverge, indicating that the cell selected the target. During FIX, firing rates after the onset of the white RDPs dropped slightly faster and showed a less pronounced increase after the initial dip during FIX compared with the TDT condition. Moreover, responses to contralateral and ipsilateral targets did not diverge; instead, they decreased similarly to firing rates in response to ipsilateral targets during TDT. Note that, in the text, we refer to

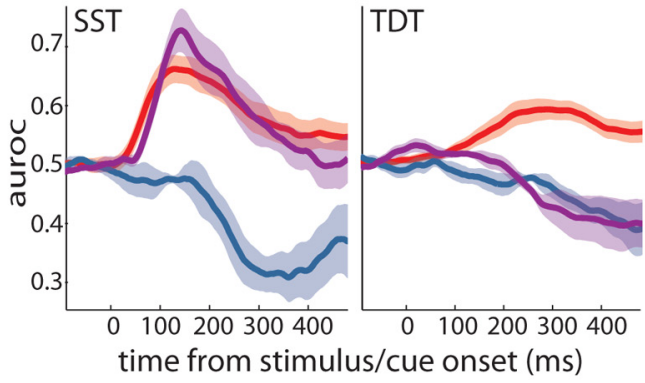

C
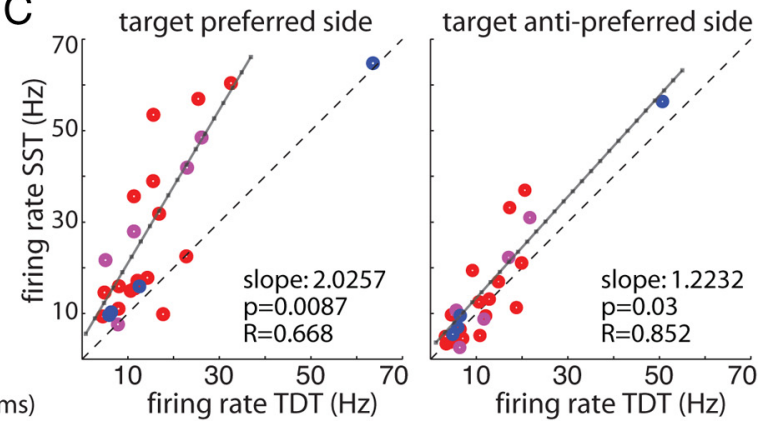

Figure 5. Modulation of target selection across tasks. $\boldsymbol{A}$, Classification of the subset of cells (total $n=25$ ) into contralateral , ipsilateral congruent (blue, $n=4)$, and contralateral incongruent (purple, $n=5$ ) based on their spatial posite side (right). Red, purple, and blue colors correspond to the respective subsets of cells as in $\boldsymbol{A}$ and $\boldsymbol{B}$. The solid black line (for details, see Results). The dashed line represents unity.

responses when the distracter was inside the RF of the MT neurons as responses to ipsilateral targets.

The average MI across selective units during TDT (solid line) and FIX (dashed line) is shown in Figure $6 B$ (left). Note that MI time courses were baseline corrected; thus, some of the values are below but not significantly different from zero. Baseline correction was applied because some units had a non-zero baseline during FIX. A likely reason for such offset from zero was the relatively small number of FIX trials $(n=\sim 36)$ (Andronache et al., 2008). During TDT, we observed a small, step-like increase in MI just after cue onset, which likely reflected the aforementioned dip in the firing rates of the single cells (also visible in the average auroc of the populations; Fig. $6 C$, solid black line). More importantly, after this initial deflection, at $\sim 300 \mathrm{~ms}$ after cue onset, target selection (MI and auroc) strongly increased and diverged significantly from zero $390 \mathrm{~ms}$ after cue onset. During FIX, the selection performance of MT remained around baseline and did not depart significantly from baseline (Fig. $6 B, C$, dashed black lines).

To compare discrimination performances in area MT and the dlPFC, we isolated MI and auroc time courses of those contralateral $(n=18)$ and ipsilateral $(n=21)$ dlPFC neurons that were recorded from the same animal (monkey Se). Figure $6 B$ (right) shows the distribution of single-cell MI latencies across cell groups during TDT. Contralateral cells (red shaded area) selected the target location significantly earlier than ipsilateral cells (blue) with an average latency of 245 and $390 \mathrm{~ms}$ from color cue onset, respectively (rank-sum test, $p=0.0031$ ). MT neurons (black) reliably selected the target $420 \mathrm{~ms}$ after cue onset. This average latency was significantly larger than that of contralateral cells $(p=0.0206)$ and similar to the latency of ipsilateral neurons $(p>0.7)$. The similarity of MT and dlPFC ipsilateral cells in their 
A

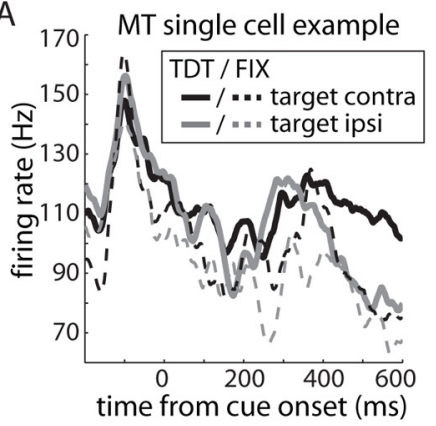

$\mathrm{B}_{10^{-2}}$

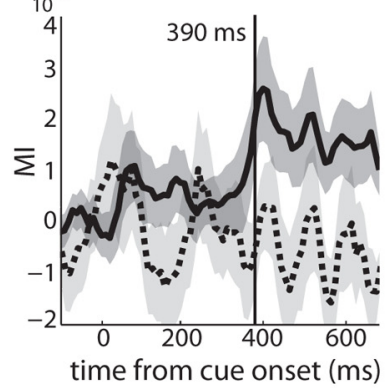

C

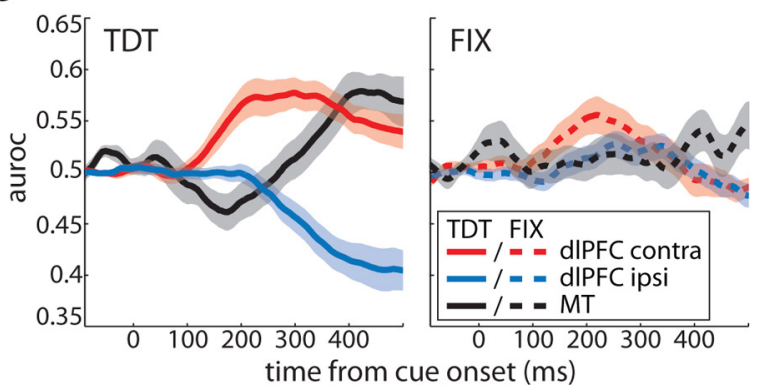

Figure 6. Target selection: dIPFC versus area MT. A, MT single-cell example. Firing rate as a function of time during TDT (solid lines) and FIX (dashed lines) for contralateral (black) and ipsilateral (gray) targets. B, Left, Average MI as a function of time from color cue onset for all target location-selective MT cells $(n=15)$ during TDT (solid line) and FIX (dashed line). Right, Distribution of selection latencies for dIPFC contralateral cells (red, $n=18$ ), dIPFC ipsilateral cells (blue, $n=21$ ), and MT cells (black). Solid vertical lines represent average selection latencies. Differences were assessed using rank-sum tests. C, Average target selection performance (auroc) as a function of time from color cue onset for the same groups of cells during TDT (left, solid lines) and FIX (right, dashed lines). All neurons were recorded in the same animal (monkey Se). Shaded areas represent the SEM. n.s., Not significant.

target selection dynamics was also reflected in the auroc time courses of the populations (Fig. 6C). During TDT, target selection evolved slowly in both groups of cells and showed similar dynamics (note that all MT cells were selective for the contralateral target, hence auroc values $>0.5$ ); during FIX when the stimuli were task irrelevant, both remained around baseline throughout the trial.

\section{Discussion}

\section{Target-distracter discrimination by dIPFC contralateral and} ipsilateral neurons

In our sample of $106 \mathrm{dlPFC}$-selective neurons, we found that target-distracter discrimination latency was significantly shorter in contralateral $(58 \%)$ relative to ipsilateral (42\%) neurons. This indicates that contralateral cells signaled the allocation of attention well before ipsilateral ones. In addition, contralateral neurons performed the target-distracter discrimination during both TDT and FIX, whereas ipsilateral neurons only did so during TDT. The latter finding suggests that contralateral neurons could discriminate targets from distracters in the absence of any modulation in the firing rate of ipsilateral neurons, at least during the time period shortly after the color change onset. Altogether, our results argue against the hypothesis that, during the allocation of attention, target-distracter discrimination across different hemifields requires competitive interactions between contralateral and ipsilateral neurons.

It is possible that discrimination by contralateral cells during FIX reflect automatic, mandatory top-down target selection that may have developed in this group of neurons through extensive training of the animals in the TDT task (Baluch and Itti, 2011). At least two hypotheses may explain why ipsilateral neurons did not

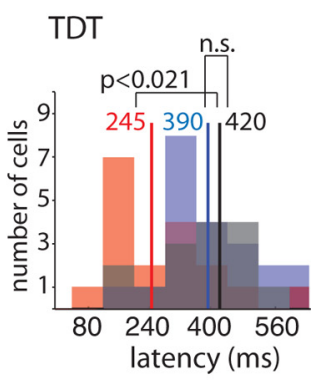

discriminate the target during FIX trials. First, it is possible that they were not affected by training in the same manner as contralateral cells. Second, it is possible that, in FIX trials, the animals automatically selected the target quickly after the color cue onset and then rapidly reallocated attention to the fixation point to detect the color change, which was the behaviorally relevant event. Because target-distracter discrimination developed later during the trial in ipsilateral neurons, there may have been simply not enough time for this process to develop in these units.

Based on the latter, one may further hypothesize that contralateral and ipsilateral neurons are associated with two sequential operations of attention. Contralateral cells may be implicated in the initial allocation of attention shortly after cue onset, as well as in the subsequent sustaining of attention on the target. Conversely, ipsilateral cells may be primarily implicated in the sustaining of attention on the target while suppressing the distracter. Albeit far reaching, this hypothesis is supported by work demonstrating that some individuals with attentional deficit and hyperactivity disorder can allocate attention to a target in the presence of distracters but are unable to sustain attention on the target (Manly et al., 2001). Thus, at the level of behavior, allocation and sustaining of attention can be dissociated. Additional studies are needed to investigate this issue.

\section{Competitive interactions in dIPFC neurons}

One interesting finding in our study was that the bias in favor of contralateral relative to ipsilateral neurons ( 58 vs $42 \%$ ) was accentuated during SST ( 85 vs $15 \%$ ) when no distracter stimulus was present. These results may explain why some studies in this area have reported contralateral cells to be $\sim 70-80 \%$ of the selective neurons (Funahashi et al., 1989, 1990; Rao et al., 1999), whereas others report $\sim 60 \%$ (Boch and Goldberg, 1989; Sakagami and Niki, 1994; Rainer et al., 1998; Everling et al., 2002). It is very likely that the number and type of dIPFC neurons that actively engage in a task also depends on additional factors, such as task difficulty, timing, attentional load, and number of distracters among others.

Importantly, during SST, only half of the neurons selective during TDT reliably discriminated the target from the distracter ( 25 of 47$)$. Of these, most were contralateral neurons. The majority of the 22 remaining units that did not discriminate the target position during SST were ipsilateral during TDT. These results indicate that many contralateral neurons can be activated by the presence of a single stimulus; thus, they may be targeted by visual inputs coming from extrastriate areas or the parietal lobe (Goldman-Rakic and Schwartz, 1982). Conversely, many ipsilateral neurons only show response modulation when the animals engage in targetdistracter discrimination. The latter, together with the lack of 
response modulation during FIX, suggest that activation of ipsilateral neurons is strongly task dependent.

Interestingly, the difference in discrimination latency between contralateral and ipsilateral neurons observed during TDT was preserved during SST. However, the amount of MI carried by both cell types significantly decreased from the SST to the TDT. This was attributable to a suppression of responses to the target at the preferred location of the neurons by the presence of the distracter in the opposite hemifield. This result strongly suggests that the distracter triggered competitive interactions similar to the ones seen in normalization circuits in visual areas during attentional tasks (Reynolds et al., 1999; Khayat et al., 2010) as well as in area LIP (Louie et al., 2011). The latter study has proposed that response normalization may be a widespread phenomenon operating in different brain areas under different task conditions.

The mechanism within the dlPFC circuitry underlying competitive interactions may be reciprocal inhibition between pyramidal cells of opposite preferred locations (Goldman-Rakic, 1995). According to that scheme, pyramidal cells encoding the contralateral and ipsilateral visual fields communicate via inhibitory interneurons. Thus, a contralateral pyramidal cell responding to a contralateral target inhibits ipsilateral pyramidal cells responding to distracters and vice versa. However, our findings argue against direct competition between contralateral and ipsilateral cells within the same hemisphere (see section above), yet the proposed hypothesis may apply when constrained to the competition between contralateral cells across hemispheres. Once the initial competition between contralateral cells of both hemispheres is resolved, the strongly active pool of contralateral neurons of the hemisphere opposite to the target may then inhibit ipsilateral cells nearby; likewise, the weakly active pool of contralateral cells in the opposite hemisphere would fail to inhibit nearby ipsilateral cells, causing increased discharge in the latter. This may explain the pattern observed in our data.

Interestingly, a previous study has provided evidence in favor of interhemispheric competition during attentional tasks (Sylvester et al., 2007). They reported that the predictability of attention-related blood oxygenation level-dependent activity in frontoparietal areas was greatly increased by subtracting the activity of homologous left and right hemispheric regions. The authors argue that the activations in the two hemispheres showed strong positive trial-to-trial correlations; differencing the signals eliminated these noise correlations and led to an efficient coding of the locus of attention. Interhemispheric difference signals have also been observed with electroencephalography (Thut et al., 2006) and single-cell recordings in monkeys (Bisley and Goldberg, 2003). One possibility is that interhemispheric interactions between contralateral neurons are implemented through the corpus callosum or subcortical pathways (Innocenti, 2009).

\section{Relationship between dIPFC and MT neurons}

We recorded the responses of a small group of MT neurons in one of the animals. As anticipated, all units had contralateral RFs and therefore can be considered in that respect similar to contralateral dlPFC neurons. Conversely, MT units did not select the target during FIX, and thus in this respect were similar to dlPFC ipsilateral neurons. The time at which MT neurons discriminated targets from distracters was substantially delayed relative to contralateral and slightly delayed relative to ipsilateral dlPFC neurons. These results are very unlikely to reflect an idiosyncratic behavior of our sample of MT neurons in this animal because the attentional modulation of responses described here has a similar intensity and time course as the one described in a previous study in area MT using a similar stimulus configuration and a task that only differed from the one of the present study in the type of cue that signaled the target location (Khayat et al., 2010).

The longer discrimination latency in MT relative to prefrontal neurons agrees with previous studies reporting longer latencies for the effects of attention in visual areas such as V4 relative to prefrontal areas (Buffalo et al., 2010). The lack of target-distracter discrimination during FIX and the $\sim 175$ longer latency in MT neurons relative to dlPFC contralateral neurons renders topdown signals sent from the latter toward the former very unlikely, at least during the initial period of target selection. Interestingly, the lack of target-distracter discrimination during FIX was shared by MT and dlPFC ipsilateral neurons. Their latency difference was $30 \mathrm{~ms}$, with ipsilateral dlPFC neurons selecting the target first. The latter findings may indicate that MT and dlPFC ipsilateral neurons are either directly inter-related or indirectly through a common signal source.

The details of the interactions between dIPFC and MT neurons cannot be elucidated with our dataset. However, one possible scenario is that signals emanating from dlPFC ipsilateral neurons target normalization circuits in MT. Increased normalization strength implemented through feedback signals would result in inhibition of responses to ipsilateral distracters relative to contralateral targets, whereas decreases in the normalization strength would result in the opposite effect (Reynolds et al., 2000; Martínez-Trujillo and Treue, 2002; Lee and Maunsell, 2009; Reynolds and Heeger, 2009). A recent study has shown that inactivation of subcortical structures thought to be involved in attentional modulation such as the superior colliculus disrupts performance in attentional tasks but leaves attentional modulation in visual area MT intact (Zénon and Krauzlis, 2012). This finding may emphasize the role of corticocortical feedback in the top-down attentional modulation of responses in visual neurons.

\section{References}

Alexander GE, Crutcher MD (1990) Functional architecture of basal ganglia circuits: neural substrates of parallel processing. Trends Neurosci 13:266271. CrossRef Medline

Andronache A, von Siebenthal M, Székely G, Cattin P (2008) Non-rigid registration of multi-modal images using both mutual information and cross-correlation. Med Image Anal 12:3-15. CrossRef Medline

Baluch F, Itti L (2011) Mechanisms of top-down attention. Trends Neurosci 34:210-224. CrossRef Medline

Barbas H, Mesulam MM (1981) Organization of afferent input to subdivisions of area 8 in the rhesus monkey. J Comp Neurol 200:407-431. CrossRef Medline

Barbas H, Henion TH, Dermon CR (1991) Diverse thalamic projections to the prefrontal cortex in the rhesus monkey. J Comp Neurol 313:65-94. CrossRef Medline

Ben Hamed S, Duhamel JR, Bremmer F, Graf W (2001) Representation of the visual field in the lateral intraparietal area of macaque monkeys: a quantitative receptive field analysis. Exp Brain Res 140:127-144. CrossRef Medline

Bisley JW, Goldberg ME (2003) Neuronal activity in the lateral intraparietal area and spatial attention. Science 299:81-86. CrossRef Medline

Blatt GJ, Andersen RA, Stoner GR (1990) Visual receptive field organization and cortico-cortical connections of the lateral intraparietal area (area LIP) in the macaque. J Comp Neurol 299:421-445. CrossRef Medline

Boch RA, Goldberg ME (1989) Participation of prefrontal neurons in the preparation of visually guided eye movements in the rhesus monkey. J Neurophysiol 61:1064-1084. Medline

Boussaoud D, Wise SP (1993) Primate frontal cortex: neuronal activity following attentional versus intentional cues. Exp Brain Res 95:15-27. CrossRef Medline

Buffalo EA, Fries P, Landman R, Liang H, Desimone R (2010) A backward progression of attentional effects in the ventral stream. Proc Natl Acad Sci U S A 107:361-365. CrossRef Medline 
Buschman TJ, Miller EK (2009) Serial, covert shifts of attention during visual search are reflected by the frontal eye fields and correlated with population oscillations. Neuron 63:386-396. CrossRef Medline

Busse L, Katzner S, Treue S (2008) Temporal dynamics of neuronal modulation during exogenous and endogenous shifts of visual attention in macaque area MT. Proc Natl Acad Sci U S A 105:16380-16385. CrossRef Medline

di Pellegrino G, Wise SP (1993) Effects of attention on visuomotor activity in the premotor and prefrontal cortex of a primate. Somatosens Mot Res 10:245-262. CrossRef Medline

Everling S, Tinsley CJ, Gaffan D, Duncan J (2002) Filtering of neural signals by focused attention in the monkey prefrontal cortex. Nat Neurosci 5:671-676. CrossRef Medline

Funahashi S, Bruce CJ, Goldman-Rakic PS (1989) Mnemonic coding of visual space in the monkey's dorsolateral prefrontal cortex. J Neurophysiol 61:331-349. Medline

Funahashi S, Bruce CJ, Goldman-Rakic PS (1990) Visuospatial coding in primate prefrontal neurons revealed by oculomotor paradigms. J Neurophysiol 63:814-831. Medline

Goldberg ME, Bushnell MC (1981) Behavioral enhancement of visual responses in monkey cerebral cortex. II. Modulation in frontal eye fields specifically related to saccades. J Neurophysiol 46:773-787. Medline

Goldman-Rakic PS (1995) Cellular basis of working memory. Neuron 14: 477-485. CrossRef Medline

Goldman-Rakic PS, Porrino LJ (1985) The primate mediodorsal (MD) nucleus and its projection to the frontal lobe. J Comp Neurol 242:535-560. CrossRef Medline

Goldman-Rakic PS, Schwartz ML (1982) Interdigitation of contralateral and ipsilateral columnar projections to frontal association cortex in primates. Science 216:755-757. CrossRef Medline

Huk AC, Shadlen MN (2005) Neural activity in macaque parietal cortex reflects temporal integration of visual motion signals during perceptual decision making. J Neurosci 25:10420-10436. CrossRef Medline

Innocenti GM (2009) Dynamic interactions between the cerebral hemispheres. Exp Brain Res 192:417-423. CrossRef Medline

Jacobson S, Trojanowski JQ (1977a) Prefrontal granular cortex of the rhesus monkey. I. Intrahemispheric cortical afferents. Brain Res 132:209-233. CrossRef Medline

Jacobson S, Trojanowski JQ (1977b) Prefrontal granular cortex of the rhesus monkey. II. Interhemispheric cortical afferents. Brain Res 132:235246. CrossRef Medline

Jones EG, Powell TP (1970) An anatomical study of converging sensory pathways within the cerebral cortex of the monkey. Brain 93:793-820. CrossRef Medline

Khayat PS, Niebergall R, Martinez-Trujillo JC (2010) Attention differentially modulates similar neuronal responses evoked by varying contrast and direction stimuli in area MT. J Neurosci 30:2188-2197. CrossRef Medline

Lee J, Maunsell JH (2009) A normalization model of attentional modulation of single unit responses. PLoS One 4:e4651. CrossRef Medline

Lennert T, Martinez-Trujillo J (2011) Strength of response suppression to distracter stimuli determines attentional-filtering performance in primate prefrontal neurons. Neuron 70:141-152. CrossRef Medline

Leon MI, Shadlen MN (2003) Representation of time by neurons in the posterior parietal cortex of the macaque. Neuron 38:317-327. CrossRef Medline

Li X, Kim B, Basso MA (2006) Transient pauses in delay-period activity of superior colliculus neurons. J Neurophysiol 95:2252-2264. CrossRef Medline

Louie K, Grattan LE, Glimcher PW (2011) Reward value-based gain control: divisive normalization in parietal cortex. J Neurosci 31:10627-10639. CrossRef Medline

Manly T, Anderson V, Nimmo-Smith I, Turner A, Watson P, Robertson IH (2001) The differential assessment of children's attention: the Test of
Everyday Attention for Children (TEA-Ch), normative sample and ADHD performance. J Child Psychol Psychiatry 42:1065-1081. CrossRef Medline

Martínez-Trujillo J, Treue S (2002) Attentional modulation strength in cortical area MT depends on stimulus contrast. Neuron 35:365-370. Medline

Martinez-Trujillo JC, Treue S (2004) Feature-based attention increases the selectivity of population responses in primate visual cortex. Curr Biol 14:744-751. CrossRef Medline

Murthy A, Ray S, Shorter SM, Priddy EG, Schall JD, Thompson KG (2007) Frontal eye field contributions to rapid corrective saccades. J Neurophysiol 97:1457-1469. CrossRef Medline

Musallam S, Corneil BD, Greger B, Scherberger H, Andersen RA (2004) Cognitive control signals for neural prosthetics. Science 305:258-262. CrossRef Medline

Pandya DN, Kuypers HG (1969) Cortico-cortical connections in the rhesus monkey. Brain Res 13:13-36. CrossRef Medline

Patel GH, Shulman GL, Baker JT, Akbudak E, Snyder AZ, Snyder LH, Corbetta M (2010) Topographic organization of macaque area LIP. Proc Natl Acad Sci U S A 107:4728-4733. CrossRef Medline

Petrides M, Pandya DN (1984) Projections to the frontal cortex from the posterior parietal region in the rhesus monkey. J Comp Neurol 228:105116. CrossRef Medline

Rainer G, Asaad WF, Miller EK (1998) Selective representation of relevant information by neurons in the primate prefrontal cortex. Nature 393: 577-579. CrossRef Medline

Rao SG, Williams GV, Goldman-Rakic PS (1999) Isodirectional tuning of adjacent interneurons and pyramidal cells during working memory: evidence for microcolumnar organization in PFC. J Neurophysiol 81:19031916. Medline

Reynolds JH, Heeger DJ (2009) The normalization model of attention. Neuron 61:168-185. CrossRef Medline

Reynolds JH, Chelazzi L, Desimone R (1999) Competitive mechanisms subserve attention in macaque areas V2 and V4. J Neurosci 19:1736-1753. Medline

Reynolds JH, Pasternak T, Desimone R (2000) Attention increases sensitivity of V4 neurons. Neuron 26:703-714. CrossRef Medline

Roitman JD, Shadlen MN (2002) Response of neurons in the lateral intraparietal area during a combined visual discrimination reaction time task. J Neurosci 22:9475-9489. Medline

Sakagami M, Niki H (1994) Spatial selectivity of go/no-go neurons in monkey prefrontal cortex. Exp Brain Res 100:165-169. Medline

Sato T, Schall JD (2001) Pre-excitatory pause in frontal eye field responses. Exp Brain Res 139:53-58. CrossRef Medline

Shannon C, Weaver W (1949) The mathematical theory of communication. Urbana, IL: University of Illinois.

Sylvester CM, Shulman GL, Jack AI, Corbetta M (2007) Asymmetry of anticipatory activity in visual cortex predicts the locus of attention and perception. J Neurosci 27:14424-14433. CrossRef Medline

Thompson KG, Hanes DP, Bichot NP, Schall JD (1996) Perceptual and motor processing stages identified in the activity of macaque frontal eye field neurons during visual search. J Neurophysiol 76:4040-4055. Medline

Thompson KG, Biscoe KL, Sato TR (2005) Neuronal basis of covert spatial attention in the frontal eye field. J Neurosci 25:9479-9487. CrossRef Medline

Thut G, Nietzel A, Brandt SA, Pascual-Leone A (2006) $\alpha$-Band electroencephalographic activity over occipital cortex indexes visuospatial attention bias and predicts visual target detection. J Neurosci 26:9494-9502. CrossRef Medline

Treue S, Martínez-Trujillo JC (1999) Feature-based attention influences motion processing gain in macaque visual cortex. Nature 399:575-579. Medline

Zénon A, Krauzlis RJ (2012) Attention deficits without cortical neuronal deficits. Nature 489:434-437. CrossRef Medline 